Euskal ikerketen aldizkaria | Revue d'études basques |

Revista de estudios vascos | Basque studies review

$1 \mid 1996$

Numéro I

\title{
Histoire et onomastique médiévales
}

\section{Jean-Baptiste Orpustan}

\section{OpenEdition \\ Journals}

Édition électronique

URL : http://journals.openedition.org/lapurdum/1907

DOI : 10.4000/lapurdum. 1907

ISSN : 1965-0655

Éditeur

IKER

Édition imprimée

Date de publication : 1 octobre 1996

Pagination : 199-221

ISBN : 2-84127-106-4

ISSN : $1273-3830$

\section{Référence électronique}

Jean-Baptiste Orpustan, « Histoire et onomastique médiévales », Lapurdum [En ligne], 1 | 1996, mis en ligne le 01 septembre 2010, consulté le 21 décembre 2020. URL : http://journals.openedition.org/ lapurdum/1907; DOI : https://doi.org/10.4000/lapurdum.1907 


\section{HISTOIRE ET ONOMASTIQUE MÉDIÉVALES}

\section{L'anoblissement de 128 maisons d'Arbéroue en 1435}

Texte original de la lettre de Jean II d'Aragon et Blanche de Navarre rois de Navarre. Archives générales de Navarre. Caj. $104 \mathrm{n}^{\circ} 42$. Tous les noms des maisons citées ont été mis en italique et les abréviations complétées.

Don Johan por la gracia de dios Rey de Nauarra Infant daragon et de Sicillia duc de Nemoux de Gandia de monblanc et de peynna fiel Comte de Ribagorça et seynnor dela ciudat De ballaguer - Et dona Blanca per la mesma gracia Reyna heredera del dicho Reyno duquessä delos dichos ducados del dicho contado et seynnora dela dicha ciudat de ballaguer a todos quantos las presentes letras beyran et oyran Salut.

Como arnalt seynnor dela casa de huhegun(,) per arnalt seynnor dela casa dayherre suson(,) gracia dueynna dela casa de lucua(,) pazco seynnor dela casa dayherre Juson(,) p.arnalt seynnor dela casa de harrieta(,) guilem arnalt seynnor dela casa de bidagayn(,) Johan seynnor dela casa de gellos(,) p.arnalt seynnor dela casa diriart(,) guilem seynnor dela casa de haranbilleta(,) Sanz seynor dela casa decheverce(,) pes seynor dela casa de berroeta(,) arnalton seynnor dela casa de aguerre(,) garcia seynor dela casa dechavarren(,) gaxernaut seynnor dela casa doyhanart(,) pes sanz seynnor dela casa decheverry(,) pes seynor dela casa de londayz yriart. martin seynnor dela casa de londayz $($, , gujllemo seynor dela casa dechapare(,) Arnalt seynnor dela casa daparabidart(,) martin seynor dela casa de ypuzaguerre(,) pazco seynnor dela casa de haranburu(,) gujxon seynor dela casa de mendiburu(,) Johan seynor dela casa darreguj (,) domenjon seynnor dela casa duhart(,) Johan seynnor dela casa doyharat(,) Johan seynnor dela casa diriberry(,) domenjon seynnor dela casa de aguerre(,) gujzoon seynor dela casa de mendi(,) arnalt seynor dela casa dechegoyen (,) Estevenja dueyna dela casa duhalde(,) arnalt seynor dela casa de larçabal(,) Johan Sanz seynnor dela casa de Çaualça(,) machin seynor dela casa derrecart(,) Johan seynor dela casa de larragoyen, remon sanz seynnor dela casa de sendugarat(,) Johan sanz seynnor dela casa de Sarrigayn(,) pes seynor dela casa delliçalde(,) gaxen seynor dela casa dechavazter et dechart darraydu(,) Johan seynor dela casa duhart Juson(,) sanz seynor dela casa durquiet(,) petrisanz seynor dela casa doch... (,) gaxen fijo dela casa delliçayri(,) domenjon seynor dela casa diriberry(,) et dirigoyen(,) johan seynor dela casa dissuri juson(,) et de baraceart dela parropia dayherre -

johan sanz seynor dela casa de sorçaval(,) gaxen seynor dela casa de heguj(,) martin seynor dela casa de laharraga(,) Johan sanz seynor dela casa de yriart(,) Sanz seynor de la casa de Sarria(,) domenjon seynor dela casa de garat(,) sanz seynor dela casa doyharberro - Johan sey- 
nor dela casa diriberri, sanz seynor dela casa durrutia(,) sanz seynor dela casa dechapare(,) johan seynor dela casa decheuehere(,) per arnalt seynor dela casa dirigoyen(,) johan seynnor dela casa de bidart, sanz seynor dela casa de aguerre, Remon seynor dela casa de soroeta(,) arnalt seynnor dela casa de Socobia (,) domenjon seynnor dela casa de gellos(,) arnalt seynor dela casa de mendilaharsu et perarnalt seynor dela casa de çavaroz dela parropia de yzturiz /

bernat seynor dela casa de oilloeta aguerre(,) guilelmo seynnor dela casa de elçurren (,) arnalt seynor dela casa de lacoaga(,) domenjon seynor dela casa de ganderaz mayor(,) sanz seynor dela casa de ganderaz (,) pes seynor dela casa doxacellay (,) arnalt seynor dela casa de haristoy(,) peyroch seynor dela casa de çaualça(,) johan seynnor del casa de çaualça suson(,) martin seynor dela casa de garat et de yriart(,) johan seynnor dela casa dargujoz(,) menaut seynnor dela casa de soriz(,) johan seynor dela casa doyharart(,) miguel seynnor dela casa dechagaray(,) pes de ....aa seynnor dela casa dibarrart (,) bernat seynnor dela casa diribarren et de garbissa(,) per arnalt seynor dela casa daguerre(,) gracia dueyna dela casa decheuerry(,) guilem seynor dela casa dinhabartiri(,) johan seynor dela casa dechegoyen et bertran seynor dela casa de sarrjlbe dela parropia de Sant martin.

johan sanz seynor dela casa dargayn juson(,) johan seynnor dela casa dirurita(,) arnalt seynor dela casa duhart echeverry(,) johan seynnor dela casa daguerre (,) johan seynor dela casa durrizpuru(,) bernat seynor dela casa de $m($ in $)$ che (,) Pes seynor dela casa de mendibil(,) johan seynor dela casa duhart(,) guillelmo seynor dela casa dechegoyen (,) mossen gaxernaut dissurj seynor dela casa de larrando et pazco seynor dela casa decheverce dela parropia de Sant estevan -

Johan sanz seynor dela casa de amezpilla(,) pes seynor dela casa daynciart(,) johan sanz seynor dela casa de bidart(,) antho ferranz seynor dela casa de aynciburu(,) Johan seynor dela casa de garat(,) arnalt seynor dela casa derrecart(,) gaxen seynor dela casa durrelçe(,) pes seynor dela casa de ospital(,) johan sanz seynor dela casa dirigoyen (,) johan sanz seynor dela casa decheverry (,) gracia dueyna dela casa de bizcay $($, domenjon seynor dela casa de baraçeart(,) Johan seynor dela casa de heguye et de Uhalde(,) Johan seynnor dela casa de heguye suson(,) lorenz seynor dela casa dechauarne(,) johan seynor dela casa diriart(,) johan seynor dela casa deyrigoyen (,) johan seynor dela casa de larrateguy(,) johan sanz dela casa diribarren decheverry et de $b$...dart $($,$) .... seynor dela$ casa de lohigorri et maria dueyna dela casa de gortayri dela parropia de helleta .

Johan seynor dela casa decheverry et de yrigoyen(,) Remon seynor dela casa diriart(,) gujxon seynor dela casa dechenjque et pes seynor dela casa daguerre dela parropia de bildarrayz

vezinos et moradores enla nuestra tierra darberoa en su tiempo y sus predeçessores seynores delas sobre dichas casas come fuyo de grant tiempo en aqua ayan usado et acostumbrado de pagar anos en nuestro tiempo et anuestros predeçessores Reyes de Nauarra quj por tiempo han seydo enl suyo por manera de cens o de fius o de pecha cada seys 
sueldos quoatro dineros de carlines prietos en cadaun aynno por cada seynor delas sobre dichas casas et de cadauna deillas - dar et pagar aqueillos por el dia et fiesta de Sent miguel de mes de Septiembre en cadaun aynno. Et mas el seynor dela sobre dicha casa de barrenuhart quj apresent est et los seynores dela sobre dicha casa quj por tiempo han seydo. El seynor dela sobre dicha casa durqujet quj apresent es et los seynores dela sobre dicha casa quj por tiempo han seydo (,) el seynnor dela sobre dicha casa delliçayri que a present es et los seynores dela dicha casa quj por tiempo han seydo cada otros vj sueldos iiij dineros dela dicha moneda . el seynor dela sobre dicha casa dechart quj apresent es et los seynores dela dicha casa quj por tiempo han seydo quoatro dineros.... poco mas omenos - El seynor delas sobre dichas casas diribarren et de yrigoyen de yssuri juson et dechart dela dicha parropia dayherre quj apresent son et por tiempo han seydo sendas goallinas et los seynores dela sobre dichas casas de yribarren de garbissa de aguerre de Jnhabartiri dechegoyen et de Sarrilbe dela dicha parropia de Sant martin sendas gaillinas et se porcieillos et corderos han sendos porcieillos et sendos corderos . et el seynor dela sobre dicha casa decheverçe dela dicha parropa de Sant esteban quj apresent es Et los seynores dela dicha casa decheverçe quj por tiempo han seydo una gaillina et si porcieillos et corderos avjan un porcieillo et un cordero pagaderos estos deueres porel dia et fiesta de navidat o enbiron en cadaun aynno anos en nuestro tiempo et anuestros predeçessores de suyo. Et que de pues en aqua que los sobre dichos deueres tornados açens fius opecha que començaron pagar los sobre dichos seynores delas sobre dichas casas et de cadauna deillas quj a present son et los quj por tiempo han seydo han usado et acostumbrado de pagar en cadaun aynno - por los dichos dias et fiestas de Sant miguel et de Navjdat o enbiron en cadaun aynno ensenble conlos fruitos delas diezmas delas dichas parropias de ayherre et de yzturjz anos en nuestro tiempo et a nuestros predeçessores Reyes de Nauarra quj por tiempo han seydo. Et que los sobre dichos deueres ensenble conlos dichos fruytos delas sobre dichas diezmas delas sobre dichas parropias dayherre et de yzturiz nuestro recebidor de nuestra tierra de ultra puertos quj a present es et los Recebidors dela dicha tierra quj por tiempo han seydo de nuestro mandamiento et de nuestros dichos predeçessores Reyes quj por tiempo han seydo de largo tiempo en aqua han usado et acostumbrado de Rentar los dichos deueres fius o pechas ensenble conlos dichos fruytos delas ditas diezmas como dicho es - por la Suma de dozientas quoranta libras dela dicha moneda poco mas o menos en cadaun aynno et por menor Suma los de mas aynnos -

Otrossi como tiempo ha que pe de billaua procurador patrimonial del Rey nuestro muy caro seynor et padre aquj dios perdone dio açens otributo perpetuo la plaça o casal entonz clamada çauaroz apresent casa hedifficada conlos heredamientos et drechos ala dicha casa pertenescientes maguer entierra de fijos dalgo por la Suma de seys sueldos quoatro dineros dela dicha moneda de cens otributo per cada un aynno et mas los fruytes delas diezmas dela heredades dela dicha casa 
pertenescientes dar et pagar las dichas diezmas et los ditos vj s. iiij d. de çens otributo al dicho procurador patrimonjal que entonz hera et por tiempo sera a pes de belçunçe bezino de nuestra dita tierra darberoa - Et que por muert del dicho pes la dicha casa de çauaroz et sus drechos et pertenencias de heredades tiene et possedesce per arnalt su fijo - Et que tiene atributo los fruytes et diezmas de las heredades ala dicha casa pertenescientes de largo tiempo en aqua por mano de nuestro amado et fiel procurador patrimonjal martin de billaua por la Suma de cinquanta sueldos dela dicha moneda apagar aqueillos ensenble conlos dichos vj s. iiij d.de çens otributo perpetuo que por todo monta ij libras .... dela dicha moneda en cadaun aynno pagaderes porel dito dia et fiesta de sant miguel de mes de septembre cadaun ayno (-)

deque nos suplicauan et pidian por merçe los sobre dichos arnalt seynor del sobre dicha casa de huhegun et los otros seynores delas sobre dichas casas ensegujent sobre nombradas Et el dicho per arnalt seynnor apresent dela sobre dicha casa de çauaroz et cada uno deillos que sobre esto de nuestra benjgna gracia et merce deynassemos enfranqujr et qujtar las sobre dichas casas et alos dichos seynnores de aqueillas quj apresent son et alos seynnores quj por tiempo seran delas dichas casas las sobre dichas cargas delos sobre dichos \& nombrados deueres fius opechas delos dichos dias de Sant miguel et de Nauidat sobre nombradas et tornar et Reincsribir las en liuertat et franqueza de jnfançonja et de fidalguja - de manera que de aqueillas puedan gozar aprouechar sen Reprension et Reproch de los dichos deueres fius o pechas sobre nombradas et mayorment que dizian que esto podiamos bien fazer sin dimjnution dela dicha Suma dela ${ }{ }^{c}{ }^{c}$ l. libras del dicho tributo delos dichos fruytos delas dichas diezmas et deuers fius opechas - los quoalles ditos fruytos delas dichas diezmas sen los dichos deueres fius o pechas faillaran agora et alos tiempos albenjr de tributo las dichas $\mathrm{ij}^{\mathrm{c}} \mathrm{xl}$. libras dela dicha moneda et en tal manera que en caso que el dicho nuestro Recebidor quj apresent es de nuestra tierra de ultra puertos et por tiempo sera non podiesse faillar mjenor que las dichas diezmas fincaran pora nos et pora nuestros sucessores Reyes de Nauarra que por tiempo seran que diesse de tributo por aqueillas las dichas ijc xl. libras Que enel dicho caso los dichos seynores delas dichas casas quj apresent son et por tiempo seran tomaran atributo las dichas diezmas por las dichas dozientas quoaranta libras et pagaran aqueillas al dicho Recebidor por manera de tributo et se gozaran et aprouecharan delas dichas diezmas Esso mesme el dicho perarnalt nos suplicaua et pidia por merçe que eil en su tiempo et sus suçessores oujentes causa de ser seynores dela dicha casa de çauaroz cargando sobre el tributo dela dicha diezma dela dicha casa de çauaroz et de sus heredades et drechos adaqueilla pertenescientes assaber ets sobre los sobre dichos Cinquoanta sueldos. los dichos seys sueldos quoatro dineros del dicho çens etributo perpetuo acomplimiento. delos dichos cinquoanta et seys sueldos quoatro dineros le quisiessemos qujtar et enfranqujr la dicha casa delos dichos vj sueldos iiij dineros por aynno / En esta forma que en caso que los fruytos dela diezma dela dicha casa el dicho procura- 
dor patrimonjal non podiere tributar por los dichos xlvj sueldos el dicho perarnalt en su tiempo et sus suçessores et herederos et obients causa que por tiempo seran seynores dela dicha casa de cauaroz enel suyo sean tenjdos de pagar por tributo dela dicha diezma los dichos lvj. sueldos iiij. dineros dela dicha moneda et gozar et aprouechar s....diezma por causa del dicho tributo - Et si el dicho procurador quj apresent es et por tiempo sera podieren tributar por mayor Suma delos dichos lvj. sueldos iiij. dineros los fruytos dela diezma dela dicha casa de çauaroz por aynno que aqueillo sea en su obcion et lo pueda fazer - Et si los dichos recebidor quj apresent es. et los quj por tiempo seran puedan tributar los dichos fruytos delas dichas parropias de ayherre et de yzturjz por mayor precio delas dichas dozients quoaranta libras dela dicha moneda por aynno et que aqueillos dichos procurador patrimonjal et Recebidor quj apresent son et por tiempo seran puedan fazer anuestro prouecho et utillidat et de nuestros suçessores Reyes de Nauarra quj por tiempo seran (-)

et Nos quj todo siempre beillamos enel prouecho et utillidat de nuestros subditos et naturales queriendo los doctar de franquezas et liuertades quanto buenament podemos inclinados ala humjl suplicacion anos feyta porel dito arnalt seynnor dela dicha casa de huhegun et por los otros seynores delas sobre dichas casas en segujent sobre nombradas et perarnalt seynor dela sobre dicha casa de çauaroz. et considerado que los sobre dichos suplicants son .... enla frontera delabort et en otras fronteras conlos quoalles frontalleros de cada dia han grands affruents por nuestro seruicio et por goardar la honor prouecho de nuestro Regno - Por las quoalles cosas sobre dichas et otras son dignos de ser liuertados et puestos por nos en honor et franqueza de fidalguia / Por esto oujdo Informacion delas cosas sobre dichas de nuestros gentes de comptos - Los quoalles bissitados los liures dela dicha cambra nos han feyto Relacion - que considerado el seguramjento que los dichos suplicantes han offrescido fazer de tener en pie el tributo delas dichas diezmas enla dicha suma delas dichas .ijcxl. libras et .lvij so. iiij dineros como ay........ que aqueilla .... en prouecho et seguridat delas dichas diezmas et de nuestro patrimonjo Real - Et que por esto las cosas en este present carta de enfranqujmjento priuillegio et liuertat contenjdas podiamos bien fazer entanto por consideracion delas cosas sobre dichas como en otras que .... auemos seydo mouidos - de nuestra cierta sciencia propria moujmjento et autoridat Real.

Alos sobre dichos arnalt seynnor dela dita casa de huhegun et alos otros seynnores delas sobre dichas casas en segujent sobre nombradas et acadauno deillos et sus herederos et suçessores quj por tiempo seran seynnores delas dichas casas et de cada una deillas perpetualment auemos enfranqujdo qujtado et Remetido - Et por thenor destas presentes - enfranqujmos qujtamos et Remetemos los sobre dichos por .... iiij. dineros deujdos por eillos et cada uno deillos como dicho es porel dia et fiesta de Sant miguel del mes de septembre en cadaun aynno - Et bien assi alos sobre dichos seynnores delas sobre dichas casas de barren uhart(,) urquiet(,) elliçayri(,) echart(,) irjbarren(,) yrigoyen(,) yssurj 
juson et baraceart dela dicha parropia dayherre (,) bernat seynnor dela dicha casa diribarren et de garbissa perarnalt seynnor dela dicha casa daguerre guilelmo seynor dela dicha casa de jnhabartiri et johan seynnor dela dicha casa dechegoyen et bertran seynnor dela dicha casa de Sarrilbe dela parropia de Sant martin et pazco seynnor dela dicha casa decheverçe dela dicha parropia de Sant esteuan et asus herederos et suçessores que por tiempo seran seynnores delas dichas casas et de cadauna deillas -

Ossi bien auemos enfranqujdo qujtado et Remetido - Et por thenor destas mesmas presentes Enfranqujmos qujtamos et Remetemos perpetualment los dichos cada otros. vjs. iiij d. dela dicha moneda porcieillo et cordero gallimas et cosas por eillos et cadauno deillos enla manera et forma sobre dicha deujdos porel dia \& fiesta de Nauidat como dicho es de susso - Et bien assi el dicho perarnalt seynor dela dita casa de fauaroz et asus herederos et suçessores seynnores que por tiempo seran dela dicha casa perpetualment auemos enfranqujdo qujtado et Remetido. Et por thenor destas mesmas presentes enfranqujmos qujtamos et Remetimos los dichos seys sueldos quoatro dineros de çens otributo por eil al dicho nuestro procurador patrimonjal por causa de nos en cadaun aynno por el dicho dia de Sant miguel por la dicha causa deujdos como dicho es -

Et aeil et atodos los otros suplicantes sobre nombrados et acadauno deillos auemos enfranqujdo qujtado et Remetido - Et por thenor destas mesmas presentes enfranqujmos qujtamos et Remetimos perpetualment - Tanto los dichos deueres fius opechas çens otributo et asas sobre dichas como todos et quoallesqujere otros drechos deueres seruitudes que por las causas et Razones sobre dichas anos et anuestros suçessores seran o podrian ser tenjdos como lauradores et por causa delas sobre dichas cargas et quoallesqujere otras como dicho es - En ultra ampliando nuestra dicha gracia enfranqujmjento qujtamjento Remjssion et libertat - auemos priuillegiado et liuertado ordenado declarado \& mandado - Et por thenor desta nuestra present carta de priuilegio franqueza et liuertat prjuilegiamos et liueramos alos sobre dichos nuestros subditos suplicantes seynnores delas dichas casas et de cadauna deillas quj apresent son et alos que por tiempo seran seynnores delas sobre dichas casas - Et ordenamos declaramos \& mandamos que eillos et sus suçessores seynores delas sobre dichas casas \& de cadauna deillas perpetualment non obstant las sobre dichas cargas deuers fius o pechas . cens otributos, et quoallesqujere otras cargas ayan de ser et sean infançones et fijos dalgo et ayan de gozar et gozen de todas las franquezas liuertades preRogatiuas et drechos con sus dichas casas sus heredamjentos et termjnos. Segunt que los otros fijos dalgo et jnfançones de nuestra dicha tierra et de todo nuestro Regno - gozan et aprouechan et las dichas casas sus heredamjentos et termjnos ayan de ser et sean francos qujtos et sueltos de toda manera de pecha carga \& serujtut por la forma \& manera que los otros dichos fijos dalgo et jnfançones dela dicha tierra de arberoa et de nuestro dicho Regno sus casas heredamjentos et termjnos han \& son francos et qujtos non obstant los 
dichos deuers fius o pechas çens otributos et otros quoallesqujere cargas como dicho es pora fazer los dichos seynnores delas dichas casas et sus suçessores que por tiempo seran seynores de aqueillas et de los dichos sus heredamjentos et termjnos sus proprias uolluntades ... cadaun d.. delos suyos francos sueltos et qujtos non obstant los dichos deuers fius opechas çens otributo como dicho es (.)

Et por thenor destas dichas mesmas presentes o coppia o uidimus deillas fecha endeujda forma - mandamos anuestros amados et fielles las gentes oydores de nuestros comptos procurador patrimonjal . martin de billaua Recebidor de nuestra tierra de ultra puertos michellot de saut et a cadauno deillos que todos et quoallesqujere lineas o lengoages que sean enlos libros de Reçeptas et expensas de nuestra dicha cambra de comptos et delos dichos procurador patrimonjal et Recebidor toquat ofazientes mencion delos sobre dichos deuers fius opechas çens o tributos sobre dichos cançellen et barren delos dichos libros et de cadauno deillos de manera que daquj adelant los sobre dichos seynnores delas dichas casas quj apresent son nj los seynnores delas dichas casas quj por tiempo seran non se faillen nj sean contreydos deuer $\mathrm{nj}$ ser tenjdos daquj adellant dar \& pagar cosa alguna delos sobre dichos deueres fius o pechas çens otributo en manera alguna - las quoalles cassamos barramos et anullamos perpetualment por nuestra autoridat Reallenqua - Todauez por thenor destas mesmas presentes auemos ordenado declarado proueydo \& mandado que si el dicho nuestro Recebidor quj apresent es opor tiempo sera delos fruytos delas dichas diezmas delas dichas casas delas dichas parropias dayherre et de yzturjz non podieren saubar las dichas dozientas quoaranta libras de Renta por aynno - Et al dicho nuestro procurador patrimonjal quj apresent es et el quj por tiempo sera non podieren faillar de tributo los dichos cinquoanta et seys sueldos et iiij dineros por cadaun aynno - Que en aqueil caso los dichos arnalt seynnor de Uhegun et los otros en segujent sobre nombrados et sus suçessores seynnores delas dichas casas quj por tiempo seran sean tenjdos de tomar atributo las dichas diezmas delas dichas parropias dayherre et ysturjz mientre que en nuestra mano et de nuestros suçessores Reyes de Nauarra quj por tiempo seran estoujeren et pagar porel dicho tributo de aqueillas las dichas dozientes et quoaranta libras (-) Et el dicho perarnalt seynnor dela dicha casa de f̧auaroz et los seynnores quj por tiempo seran dela dicha casa los dichos cinquoanta \& seys sueldos quoatro dineros del dicho tributo dela dicha diezma delas heredades dela dicha casa de çauaroz por cadaun aynno - Et eillos assi tributando las dichas diezmas enla manera et forma sobre dichas et pagando el dicho tributo o tributos como dicho es puedan fazer sus proprias bolluntades delas dichas diezmas mientre que tributadas las toujeren -

Empero que si por bentura por nos o por nuestros suçessores quj por tiempo seran trasportassemos otrasportassen alienassemos o alienassen las dichas diezmas por dono bienficio bendicion cambio o otrament en otra manera seria dela corona de Nauarra que en aqueil caso los sobre dichos suplicantes nj alguien deillos nj los suyos suçessores 
seynores delas dichas casas nj por tiempo seran non sean tenjdos de tributar las dichas diezmas nj algunas deillas si por su bolluntat et querer non querran - Et Rogamos JnJungimos et Requerimos anuestro muy caro \& muy amado fijo primo genjto et heredero don Karlos principe de biana - Et mandamos anuestros amados et fielles las gentes de nuestro conseillo - Alcaldes de nuestra cort gentes oydores de nuestros comptos thessorero gentes de nuestras finanças procurador patrimonjal et fiscal Recebidor de nuestra dicha tierra de Ultra puertos et atodos quoallesqujere nuestros suçessores officialles et subditos quj apresent son et por tiempo seran aquj esto toqua \& pertenesçe o podra toquar \& pertenescer que esta nuestra carta de prjuillegio enfranqujmiento quitamjento Remissjon et libertat - Et las cosas en eilla contenjdas enla forma et manera sobre dicha tengan goarden obserben et complan et fagan tener goardar obseruar \& complir Jnbiollablement aperpetuo sen poner embargo contrasto jmpediment difficultat empachamjento alguno en tiempo alguno en alguna manera - Car assi lo queremos et nos plaze non contrastant quoallesqujere nuestras ordenanças estatutos et estillos de nuestra thesoreria et cambra de comptos aesto contrarians -

Et en testimonio desto et firmeça et confirmacion delas cosas sobre dichas et cada una deillas auemos feyto sieillar les presentes en pendient de nuestro grant Sieillo dela chanceleria en cordon de seda et cera berde - Datum en Ollit so el dicho Sieyllo . xvij dia de março. Anno anatale domini mo $\operatorname{cccc}^{\mathrm{O}} \mathrm{xxx}^{\mathrm{O}}$ qujnto - Ay jnterllinjos do se leye (:) a todos quantos las presentes beran \& oyran Salut . pagadores porel dicho dia $\&$ fiesta de Sant miguel del mes de septembre en cadaun aynno como tales . Datum ut supra - ... por el Rey \& por la Reyna

Por mi Deamunuriz Secretario delos sobre dichos senyores Rey \& Reyna mios senyores et fue feyt colation dest present coppia ensemble con la letra original bien \& fielment de mot amot ...

*

\section{Le contexte historique}

Il revient aux historiens médiévistes de commenter tout ce que cet important document apporte pour la compréhension de l'ancienne société des vallées bas-navarraises à la fin du Moyen Àge et des problèmes auxquels est confrontée la monarchie navarraise des derniers temps. On se contente de signaler ici quelques repères.

Les rois de Navarre en question sont Blanche "héritière de Navarre" comme il est dit (+1441), fille et unique héritière de Charles III d'Evreux dit "le Noble" (+1425), son mari Jean "infant d'Aragon" (+1479) et roi de Navarre par son mariage, et leur fils le célèbre "prince de Viane", qui s'opposera à son père tenu pour usurpateur du trône navarrais, prisonnier de ce dernier par deux fois et mort en 1461 avant d'avoir pu régner.

Le privilège d'anoblissement accordé sur leur demande aux maîtres des maisons et leurs successeurs vient, comme l'indique le texte, au 
moment où la Basse-Navarre est entourée d'une atmosphère d'hostilités, avant la fin prochaine de la guerre de Cent Ans par la victoire des armées du roi de France sur celles du duc d'Aquitaine et roi d'Angleterre (14491450); dans le même contexte le roi d'Angleterre a nommé Louis de Beaumont connétable de Navarre et comte de Lérin, chef du parti "beaumontais" pro-castillan (et alors pro-anglais) pour gouverneur du château de Mauléon, et c'est lui qui fera tuer le "Berterretche" de la fameuse chanson, très certainement du parti "gramontais" et pro-français.

Le privilège répond aussi à un mouvement général de modernisation des anciens tributs et "devoirs" (autrefois en nature, déjà transformés en argent : le "tribut" de la terre d'Arberoue est de 25 livres dans les comptes de la fin du XIII' siècle), ici pour les maisons de laboureurs dites "franches". Ce texte, exactement comme le Censier quasi contemporain de Soule, montre les degrés de dépendance divers qu'impliquait cet ancien statut. En 1418, à la suite d'un long procès, Charles III a de même ramené à une redevance annuelle en argent les devoirs des laboureurs de la vallée d'Ossès, qui n'ont pas cependant demandé ni obtenu le statut d'infançons. Aux hostilités frontalières s'ajoutent d'autres malheurs, qui font réduire les taxes de Saint-Jean-Pied-de-Port en 1415 (brève correspondance en basque entre les responsables de Cize et de Pampelune), et celles de toutes les terres de Basse-Navarre, AinhiceMongelos étant détruite pour raison de peste, en cette même année 1435.

Ce texte souvent cité, dans le Diccionario de Antiguedades del reino de Navarra de Yanguas y Miranda publié au siècle dernier par exemple, mais jamais reproduit, n'anoblit pas "111 maisons" comme on l'a écrit, mais bien 128 .

\section{Les maisons anoblies}

Par suite de cette lettre d'anoblissement, les maîtres des maisons nommées, et de même les "maîtresses" en cas d'absence de maître, cet anoblissement par les femmes devant être aussi noté, ainsi qu'un "fils" (peut-être alors mineur ?) et leurs "héritiers et successeurs" sont tenus pour nobles, c'est-à-dire dans le vocabulaire navarrais médiéval et traditionnel "infançons et hidalgos", "perpétuellement", mais au seul titre des maisons nommées. C'est la conception ancienne de la noblesse dite "réelle" qui se poursuit : les héritier's des nouveaux nobles qui s'installeraient hors des maisons citées n'auraient pas plus le droit de se tenir ni de tenir leur domaines pour nobles que les héritiers des maisons nobles anciennes s'installant dans une maison non noble ne l'avaient. La preuve en est donnée par deux fois : pour "mossen gaxernaut dissurj", qui est, vu son titre de "monseigneur", un descendant de la maison Ixuri d'Ayherre, l'une des 7 maisons infançonnes anciennes du lieu, mais n'est anobli qu'en tant que "maître de la maison Larrando" de SaintEsteben, et plus clairement encore pour "Per Arnalt de Belzuntz", dont la filiation est établie avec la maison Belzuntz également d'Ayherre, la plus importante et la plus connue de toutes les maisons nobles 
anciennes d'Arbéroue, mais qui ne se trouve anobli que comme maître de la maison Zabarotz d'Izturitz. La filiation, si elle explique pour une part que cette maison et son maître soient tant de fois cités dans la lettre, ne jouera qu'à partir de ce Per Arnalt et ses successeurs et héritiers maîtres de Zabarotz et pour eux seuls.

La maison Zabarotz bénéficie grâce à cette lettre d'un complément d'histoire assez précis : faite en "terre d'infançon", elle avait appartenu au fameux Pes de Laxague (du nom de sa maison noble Latsaga d'Asme en Ostabarret), qui était lui-même apparenté à la famille royale par sa mère et avait épousé Jeanne de Beaumont fille naturelle de l'infant Louis de Navarre (fils de Charles II et frère de Charles III), et se nomme dans une lettre de 1391 "cavaillero et camberlenc", chevalier et chambellan (de Charles III). Il la mentionne dans son long testament rédigé peu de mois avant sa mort en février 1393 et publié en 1881 par G. Bascle de Lagrèze (La Navarre française, II, p. 426-440), avec son "moulin" et son "bois" : tot lostau, molii, bosq apperat Sabarodsa. Assez vite les biens de Pes de Laxague se trouvèrent sans héritiers et furent joints au domaine royal dès le début du siècle suivant (cf. Béatrice Leroy: "Un seigneur de Navarre à la fin du XIV siècle : Pes de Laxague", Revue de Pau et du Béarn, n¹2, 1984-85). C'est le temps où elle dut être délaissée et plus ou moins détruite, puisque lorsque Charles III l'attribue à Pes de Belzuntz, "habitant d'Ayherre" et père de Per Arnalt, c'était "une place et un casal" quoique pourvu d'un domaine et redevable de diverses taxes. Le même Charles III dans les mêmes conditions attribua, par une lettre du 3 février 1396 (en fait 1397), la maison Irumbehere d'Exave en vallée d'Ossès restée sans héritier à Bertrand de SainteEngrâce (maison noble de Juxue), lequel était précisément l'un des neveux et l'héritier principal de ... Pes de Laxague, en récompense des "bons et agréables services" à lui rendus.

Depuis peu "édifiée" en 1435, ou mieux réédifiée sur la "place" existant déjà, la maison Zabarotz porte en tout cas un nom très ancien, avec la dérivation archaïque "aquitaine" par le suffixe locatif - $O z$, indiquant une friche si le radical vraisemblable est le mot sapar "ronce", ou peut-être un terrain plat, avec une altération de $-l$ - en - $r$ - (comparable au nom du territoire, écrit tantôt Arberoa et plus souvent Arbeloa) pour le mot zabal. Le "pas de toponyme connu" d'un article déjà ancien de J. Lemoine (Bulletin de la Société des Sciences, Lettres et Arts de Bayonne, $\mathrm{n}^{\circ} 133,1977 \mathrm{p} .139$ ), pour un habitant bayonnais de ce nom à la fin du Moyen Age, demande rectification.

Ce document comporte d'autres noms de maisons absents des recensements antérieurs, notamment ceux de 1350, 1366 et 1412, publiés au Bulletin du Musée Basque ainsi que la liste complète des maisons citées. Il faudra donc compléter ainsi la liste des maisons médiévales, en supposant que, faute de précision contraire, toutes sont en principe "franches" et non "fivatières" de quelque autre maison, village par village, en orthographe basque usuelle et conforme aux formes anciennes, très soigneusement reproduites dans ce texte comme on le verra plus loin : 
- à Ayherre : Lukua, Etxabarren, Londaitz-Iriarte (indépendamment de celle dite simplement Londaitz et de la maison infançonne du même nom), Apara-Bidarte, Iputzagerre, Uharte, Oiharat, Mendi, Larzabal, Zabalza, Larragoien, Sarrigain, Elizalde, Urkieta, Elizairi, Iriberri, Irigoien, Baratzearte, ainsi qu'un nom peu lisible qui est peut-être Ochobi ;

- à Izturitz: Iriarte, Garate, Etxebehere, Irigoien, Bidarte, Agerre, Jelos ;

- à Saint-Martin : Oiloeta-agerre, Iriarte, Etxegoien, Sarrilbe ;

- à Saint-Esteben: Uharte-etxeberri, Mixe (actuellement Minche, qui suppose une petite erreur graphique en 1435), Mendibil ;

- à Hélette : Bidarte, Urrelze, Ospitale, Uhalde, Hegi-garai, Etxabarne, Iribarne, Lohigorri et un nom peu lisible commençant par $b$ - et s'achevant par-arte, mais qui n'est ni "Bidarte" ni "Baratzearte";

- à Bildarraitz (toujours compté comme un hameau et ici une "paroisse" à part au Moyen Age, aujourd'hui confondu dans Ayherre, limitrophe de Mendionde): Etxeberri, Irigoien.

Inversement, quelques maisons citées auparavant sont absentes :

- Johan de Lacoaga cité en 1350 sans autre précision portait peutêtre un nom d'origine extérieure à Ayherre ;

- Luro, Ameztoy et Etcheberrj citées en 1412 à Izturitz étaient bien en revanche des maisons avec leurs "seigneurs" ; et il faut remplacer au même lieu dans les listes précédemment publiées "Etxebertze", erreur de lecture, par Etxebehere, recensée donc en 1350, 1366, et peut-être écrite par erreur etcheberrj dans la liste approximativement datée de 1412 par les archivistes de Pampelune ;

- à Saint-Esteben Etxebertze-urrizpuru de 1366 et Apezetche de 1412 ne sont plus citées, ni les fivatiers de la "salle de Sorhaburu" Iara, Arrieta, Basagaitz, Urgaran, Etchart, qui gardent donc leur ancien statut ; il en va de même pour Méharin dont aucune des maisons, toutes reconnues fivatières de la "salle" un siècle plus tôt, n'est citée ;

- à Hélette Aguerre (différente de la "salle" noble du même nom) dont le maître était nommé en 1412 n'est plus citée, ni Urkudoy qui apparaît dans d'autres documents.

Ces différences, mineures sauf pour Ayherre, s'expliquent par le caractère incomplet de la plupart des recensements d'une part ; mais aussi par des changements réels probables : disparition de quelques maisons qui ont pu se trouver dépeuplées, à mettre en relation avec le fait que plusieurs maisons ici ou là appartiennent à un seul maître, effet des épidémies (la rémission d'impôts pour "peste" est de cette même année) ou d'autres causes; mais aussi apparition de quelques maisons plus récentes, dont témoignerait justement la réédification de Zabarotz.

Le total des maisons "nobles" d'Arbéroue, si l'on y ajoute les 17 infançons "anciens" (7 à Ayherre, 1 à Izturitz, 1 à Saint-Martin, 3 à Saint-Ešteben, 4 à Hélette, 1 à Méharin), s'élève donc à 145 à partir de 
1435. Ce chiffre, même en y ajoutant les rares fivatiers, n'atteint pas le nombre des " 200 maisons anciennes" signalé quelques siècles plus tard. Il n'a dû être fixé qu'au cours du XVI'siècle, alors qu'un mouvement de repeuplement s'est généralisé à partir du milieu du $\mathrm{XV}^{\mathrm{e}}$ siècle.

La comparaison des trois grands recensements ou "fouages" antérieurs à 1435 (cf. Bulletin du Musée Basque nºs 65, 73, 75, 76, 79, 84, 85, $87,90,105,125)$ fait apparaître une courbe descendante puis ascendante dans le nombre de feux recensés (maisons infançonnes anciennes non comprises) dans l'espace d'un peu moins d'un siècle entre 1350 et 1412 , avec des variantes dans chaque village successivement en 1350 , 1366,1412 (la date réelle est peut-être un peu antérieure) et 1435 :

- Ayherre : 46 - 25 - 20 - 46.

- Bildarraitz : 4 - 2 - 2 - 5 .

- Izturitz : $17-8-11-19$.

- Saint-Martin : $15-12-12$ - 21.

- Saint-Esteben : $12-7-8-11$.

- Hélette : 17 - 10 - 8 - 24.

Des noms qui n'apparaissaient pas en 1412 réapparaissent en 1435, l'ensemble manifestant, hors du nombre restreint de noms cités pour la première fois en 1435 (mais pas forcément nouveaux), une très forte stabilité, comme c'est la règle à peu près générale pour les maisons des territoires intérieurs.

\section{Observations onomastiques}

\section{1. Les prénoms}

Une comparaison rapide avec les noms recensés pour 1412 permet de dégager les constantes et les variations les plus nettes dans l'usage des prénoms. Cette liste de date approximative, rédigée en gascon, est la suivante village par village, les maisons nobles anciennes mises entre parenthèses (seules 4 sur 7 sont nommées à Ayherre, dans une proportion comparable au reste de l'habitat), et sans reporter les lettres usuelles de ces rôles correspondant aux degrés de richesse et de taxation $(\mathrm{R}, \mathrm{s}, \mathrm{t}, \mathrm{p}$ : "riche, second, troisième, dernier" du plus fort au plus faible); il y a un "degan", comme en Mixe depuis la réforme de 1316, qui représente chaque "paroisse" :

- Ayherre : (P(rim)o la salle debelçunçe) - johan sr detcheuerrj - Pes durruthi sr doyhanart (ce nom est répété pour deux maisons, l'une étant certainement "Oiharat" dans les autres listes) - Garchot sr de berhoete Johan sr daguerre - Toan sr detcheuerçe - bernat sr de harambilete Guillen sr detchapare - Sanz sr de gelos - Johan sr de bidegayn - bernat sr de harrjete - arnalt sr dayherre iuso - pelen sr dayherre suso - Gujllelmo sr dirjart degan - Pes $s r$ duhart - bernat $s r$ de londaytz - (la salle dissurj) Johan sr detchauazter - (la salle darraydu) - pes sr dechagoyen - Johan sr duhalde - Sanz sr darrecart - (la salle de mendigorrj) - martin faur estatjant aguerre - Petri Sanz sr doyhanart - bernat sr de haramburu - 
- Bildarraitz : Remon sr dirjart de bildarraytz - Antoquo sr daguerre de bildarraytz -

- Izturitz: P(rim)o iohan sr de sorçabau degan - Johan sr de laharrague - arnalt $s r$ de biellenaue - Sanz $s r$ detchagapare - miqueu $s r$ de mendj laharrssu - per arnalt sr de Socobie - miqueu sr de sorhoete - (la salle de satharitz) - Johan sr de luro - Johan sr duhart - per arnalt sr dameztuy - Johan sr detcheuerri -

- Saint-Martin : (P(rim)o la salle de st martin) - menaut sr diuarrart - arnalt sr de hariztuy - Pes sr dossaçelay - pes sr de gandaratz - Johan $s r$ de laquoague - arnalt sr de çabalc e iuso - arnalt sr de garat - iohan sr darrguioz - pes sr dinhauarrtyrj - Johan sr daguerre goloyotz - Johan $s r$ decheuerri de goloyotz -

- Saint-Esteben : (P(rim)o la salle de sorhaburu) - (la salle de Sant esteben) - Johan sr detchart - Guillem sr de bassagaytz - Pes sr durquaren (ces trois dernières maisons sont dites "questaux de la salle de Sorhaburu") - pes sr detcheuerçe de larrondo - per arnalt sr dapezetche per arnalt sr darrgayn - bernat sr durrizburu - per arnalt sr dirurjta -

- Hélette : (P(rim)o la salle de garre suson) - (la salle de garre iuso) - Gassernaut de baratçeart - Enecossanz estatgant de Sante marie (c'est un "locataire" de la maison noble Sainte-Marie) - Sanz de heguie - arnalt sanz sr de heguie (il y a effectivement deux maisons de ce nom à Hélette) - arnalt estatjant yrjart - Guillelmo sr de larrategui - Petyrj sr daguerre (uchusco sr daguerre de helete) - Garatea - Errecartea -

Un premier élément de comparaison touche la répétition des noms, qui révèle l'identité des maîtres d'une même maison à 23 ans (et probablement un peu plus vu la date incertaine de 1412) de distance. Il est clair que peu des maîtres de 1412 ont survécu jusqu'en 1435, à se fier du moins à l'identité des prénoms, elle-même un peu incertaine vu la fréquence des prénoms qui se répétaient, hier comme aujourd'hui, de père en fils, et l'habitude de rappeler le nom du père en seconde position (dans les premiers temps suffixé en -iz/-ez) s'étant perdue à partir du XIII' siècle :

- un seul à Ayherre : guillelmo de Etchapare ;

- un aussi (et la proportion de $5 \%$ passe alors à $50 \%$ !) à Bildarraitz : Remon de Iriarte ;

- trois (et peut-être quatre avec un per arnalt en 1412 et un arnalt en 1435 pour Zokobia) à Izturitz : Johan de Sorzabal (le "doyen" de 1412), Zanz de Etcha(ga)pare, Johan de Etchebehere (noté par erreur etcheberri en 1412);

- trois à Saint-Martin : Arnalt de Hariztoy (le maître de cette maison qui était "alcalde" ou juge d'Arbéroue en 1350 portait le même prénom, mais ce n'était assurément pas le même !), Pes d'Otsazelhai, Johan d'Argiotz. Sur 53 maisons identiques nommées en 1412 et 1435, les personnages de même nom, au nombre de 8 , forment $15 \%$. 
La valeur des comparaisons portant sur la fréquence des prénoms employés en 1412 et 1435 ne peut être qu'approximative et très peu ou inégalement révélatrice sur les usages réels, surtout pour les noms n'apparaissant qu'à un petit nombre d'exemplaires. Elle devient plus significative pour ceux qui ont une haute fréquence dans chaque liste.

L'ordre de fréquence pour le total de 63 prénoms cités en 1412 , donne du plus grand nombre au plus petit :

- pour les noms simples : 15 Johan soit près de $24 \%$, 10 Pes et Petiri (cette adaptation basque n'apparaît qu'une fois) ensemble près de $16 \%$, 5 Guillem/Guillelmo près de $8 \%, 5$ Bernat idem, 4 Arnalt plus de $6 \%, 4$ Sanz idem, 3 Miguel/Miqueu $4,8 \%$; et à un seul exemplaire : Antoquo (diminutif basque de l'ancien Anto), Garchot (diminutif à palatalisation basque et suffixe roman de Garcia), Martin, Menaut, Toan (ce prénom cité quelquefois et qui ne peut être confondu avec "Johan" est peut-être l'un des nombreux diminutifs du répandu "Arnaut" dont les formes sont parfois inattendues), Uchusco (ce prénom-surnom basque diminutif et hypocoristique "petit vilain" est fort répandu au Moyen-Äge) ;

- pour les noms doubles : 5 Per Arnalt soit près de $8 \%$, et tous les autres à un seul exemplaire : Arnalt Sanz, Eneco Sanz (le vieux prénom local "Eneko" devient très rare), Gassernaut (phonétique totalement romane pour "Garcia-Arnalt"), Petri Sanz.

En 1435, sur les 128 maisons anoblies, 8 n'ont pas de maître propre, et comme un prénom n'est pas inscrit sur le parchemin, le calcul des fréquences porte sur 111 prénoms (et non "maisons" !), répartis ainsi du plus grand nombre au plus petit :

- pour les noms simples : 27 Johan soit plus de 24\%, 12 Arnalt (dont 1 Arnalton diminutif) soit environ de $11 \%, 9$ Pes $8 \%$, 8 Sanz 7,3\%, 7 Domenjon 6,37\%, 6 Guillelmo/Guillem 5,4\%, 5 Martin 4,5\%, 4 Gaxen (ce prénom, qui ressemble à un dérivé du "Gassie" roman issu de "Garcia", est attesté dans la région du $\mathrm{XI}^{e}$ au XIII" siècle) 3,6\%, 3 Bernat 2,7\%, 3 Guizon/Guixon (typique prénom-surnom porté depuis le Moyen Age jusqu'aux temps modernes) idem, 3 Pazco idem, 2 Remon $1,8 \%$; et à un seul exemplaire : Bertran, Garcia, Lorenz, Menaut, Miguel, Peyroch (diminutif de "Peyre" l'une des formes romanes de "Pierre" comme "Pes");

- pour les noms doubles : 8 Johan Sanz 7,3\%, 6 Per Arnalt 5,4\%, 2 Gaxernaut 1,8\%, 2 Pes/Petri Sanz idem; et un seul exemplaire de Antho Ferranz.

Les 5 prénoms féminins de 1435 ne permettent aucune comparaison : 3 Gracia (il y avait une "Guerachit daune (dame) durruthi" à Armendaritz en 1412), 1 Esteuenia, 1 Maria.

Les constantes les plus nettes touchent Johan qui nomme dans les deux cas un homme sur quatre environ, Sanz autour de 6 à $7 \%$. La fréquence de ces noms est accentuée par leur emploi en prénom double. 
Les variantes montrent la diminution importante de Pes et variantes de 16 à $8 \%$, de Bernat de 8 à $2,5 \%$, moins sensible pour Guillen et variantes de 8 à $5,4 \%$. L'augmentation apparente d'Arnalt de 6 à 10,8\% doit résulter du hasard de l'échantillonnage, car c'est un prénom de toute manière très abondant, de très loin en tête des prénoms masculins en 1305-1350, en diminution ensuite. Surtout on voit apparaître en 1435 des noms absents en 1412 et rares dans tous les documents antérieurs en zone bascophone : Pazco apparaît à 2,7 \% (trois emplois) en 1435, absent en 1412 et à 1 seul exemplaire sur 911 noms d'homme recensés en Basse-Navarre et Soule entre 1305 et 1350 (étude présentée aux journées de l'Académie basque à Estella en septembre 1990, non publiée à ce jour). Étaient aussi absents en 1412 Domenjon (diminutif roman de Domintx pour "Dominique", abonde en zone romane après 1400$) 6,37 \%$ en 1435 (5 exemplaires de même base sur les 911 noms de 1305-1350 : cf. supra), et Gaxen avec 4 exemplaires en 1435 : ce sont incontestablement des prénoms répondant à une nouvelle mode qui semble, malgré les exemples de formes basques (prénom Petiri, prénom-surnom Guixon...) du reste fort rares, procéder d'une influence romanisante.

La courbe des prénoms les plus portés, entre la période 13051350 et celle de 1412-1435, il est vrai sur des échantillons numériquement très différents, fait voir des changements significatifs dans leur mode et leur emploi, et partant dans les références socio-culturelles qui les commandent. La progression de Johan est remarquable, qui passe du $7^{\circ}$ rang avec $7 \%$ au début du XIV'e siècle au premier avec plus de $24 \%$ au début du quinzième ; il est vrai que pour la seule Arbéroue il était au premier rang en 1350 mais à égalité avec Pes et Sanz. Inversement Pes et ses variantes de la première position avec plus de $21 \%$ descendent à $16 \%$ en 1412 et $8 \%$ (pour un échantillon plus important) en 1435 ; descendent aussi Bernat de $12 \%$ ( $3^{c}$ par la fréquence en $1305-1350$ ) à $8 \%$ en 1412 et $2,7 \%$ en 1435 , et Garcia à $8 \%$ en 1305-1350 mais pratiquement absent un siècle plus tard (le cas de Gaxen à part). Sanz (dont la forme ancienne Sancho disparaît au moins dans l'usage officiel) reste inchangé entre $8 \%$ en 1305-1350 et $7,2 \%$ en 1435 , tandis que Guillem et ses variantes s'emploient de moins en moins : $9 \%$ ( $4^{\circ}$ en fréquence totale y compris en Arbéroue) au XIV ${ }^{e}$ siècle, $8 \%$ encore en 1412 (échantillon plus réduit) et seulement $5,4 \%$ en 1435 . En revanche Martin (en forme basque "diminutivée" habituellement Machin) de fréquence négligeable au début du $\mathrm{XIV}^{e}$ siècle, au $12^{e}$ rang et à $1 \%$, surtout employé chez quelques nobles, passe à $4,5 \%$ en 1435 , tandis que le vieux prénom Eneco un peu plus employé que le précédent (donc déjà rare) au XIV siècle à $1,2 \%$, mais cependant absent d'Arbéroue, y apparaît une fois au $\mathrm{XV}^{\mathrm{c}}$; mais Lup/Ochoa (1 exemplaire en Arbéroue en 1350) disparaît. Disparaissent aussi les vieux noms dits "ethniques" dont le plus notable était Navarre (masculin et féminin) déjà assez rare en 13051350 ( 4 exemplaires sur 911 pour les hommes, mais 8 sur 95 pour les femmes). 
Les doubles prénoms diminuent de moitié, puisque de ces mêmes 911 noms, 281, soit près du tiers, étaient doubles, et seulement 15 sur $110(1 / 7)$ en 1435.

La "modernisation" des prénoms se fait donc avec la disparition ou la diminution plus ou moins sensible des vieux prénoms régionaux, la progression de certains autres : Johan qui est devenu aussi prénom dynastique des rois de Navarre (Jean II, puis encore Jean III d'Albret, et Jeanne ensuite...), Martin qui doit bénéficier du vocable de la paroisse du même nom au cœur de l'Arbéroue. Pour les trois noms féminins cités, il faut remarquer que Gracia était le 4' prénom le plus employé parmi les 95 femmes recensées dans les documents et les territoires précédemment signalés en 1305-1350 à $6 \%$, Maria le $1^{\text {er }}$ à $25 \%$, mais Estevenia à un seul exemplaire.

Il y aurait à interroger enfin la mode des cultes de saints, variable selon les lieux et les temps comme on sait. Les prénoms-surnoms toujours employés au début du XV $\mathrm{XV}^{e}$ siècle, guizon, guixon, uchusco qui sont respectivement "homme, petit homme, petit vilain", indiquent que la dénomination, du moins usuelle, n'était pas encore toujours de même nature que ce qu'on entend plus tard par "nom de baptême".

\section{2. Les noms de maisons}

En toponymie, la liste des noms de maisons appelle aussi quelques observations, pour des noms presque tous cités depuis au moins un siècle à cette date de 1435, certains depuis deux siècles (guerre du roi de Navarre en Labourd de 1245-1249) et même davantage (les citations au Livre d'Or de Bayonne et au Cartulaire de Sorde commencent au XI ${ }^{\mathrm{e}}$ siècle). La "lettre" de 1435 donne l'impression générale que les noms, tous d'étymologie basque avec quelques emprunts latins déjà anciens (eliza, ospitale), certains même de la série très archaïque des dérivés en $-o z$ et $-i z$, et un seul nom incertain qui apparaît ici pour la première fois (Minche), sont transcrits avec beaucoup de soin, ce qui n'est pas rare dans ces documents des $\mathrm{XIV}^{e}$ et $\mathrm{XV}^{e}$ siècles où l'administration navarraise devait être largement bascophone, au point que les scribes parfois écrivent les noms restitués ou même décomposés : ce fait de la réécriture des noms, pour leur donner en général la forme basque la plus explicite ou la plus explicable (en 1412 on lit par exemple le groupe nom-adjectif détaché dans mendj laharrssu), oblige à ne pas déduire trop hâtivement et dans tous les cas les formes parlées ou "phonétiques" à partir des formes écrites. Une marque de cette attention est la transcription des vibrantes fortes écrites doubles à l'intérieur des mots devant consonne (exemple précédent) ou en finale de mot dans le texte de 1412.

La romanisation se voit habituellement dans les traductions des adjectifs toponymiques communs "behere, garai, handi, xipi, berri" etc. qui deviennent "iuso(n), suso(n), mayor (qui traduit aussi très souvent le gapare de Etxegapare en "Casemayor"), menor" etc... ; lorsque c'est le 
seul adjectif qui est traduit, il faut entendre qu'il s'agit d'une épithète non intégrée dans la composition, et on ne traduit pas le seul second élément de noms comme Etxeberri, Etxegoien etc... Le texte de 1435 fournit ainsi ayherre suson, ayherre Juson (en 1366 Ayherre garay, Ayherre behere), issuri juson (en 1366 Yssurj behere) etc... En effet inverse, le texte propose une fois une traduction basque d'une formule romanisée : une maison d'Ayherre est d'abord écrite uhart Juson (même formule en 1366), puis au moment de rappeler le "cens" de "six sous quatre deniers carlins" qu'elle devait traditionnellement avec d'autres au roi de Navarre, elle devient barren uhart, en antéposition archaïque d'adjectif facilitée par le degré superlatif (comme Goienetxe, Barrenetxe...). Cet emploi, qui signale peut-être aussi que le scribe ou notaire était bascophone, fait comprendre que "barren" et "behere" étaient perçus comme équivalents, même s'ils sont d'étymologie (Barretxe existe à côté de Barrenetxe) et de sens distincts : "le plus à l'intérieur" pour "barren" qui a fait le dialectal moderne "barne" ainsi noté parfois déjà au XIV" siècle et ici pour echauarne à Hélette, et "situé en bas" pour "behere" : actuellement cette maison se nomme Barne Uhartea. Dans la désignation des lieux, "bas" et "intérieur" se fondent sémantiquement par rapport aux lieux élevés et aux chemins d'accès qui servent de points de repère.

Les autres marques de romanisation sont pour la plupart d'ordre simplement phonétique et résultent d'une "officialisation linguistique" (latinisation d'abord, romanisation ensuite à partir du XIII siècle) des noms basques qui remonte aux origines mêmes de la tradition écrite des chancelleries, et qui se retrouve aujourd'hui dans un grand nombre de noms de lieux ou de patronymes d'état civil (issus des noms de maisons). On peut relever ainsi :

- la chute du -e final de arte devenu atone en phonétique romane dans iriart, uhart, oyharart, bidart, errecart, tandis que alde de uhalde ne perd -e que si l'ensemble du mot est romanisé (uhaut est alors la forme romane très fréquente au XIV siècle, mais inconnue des locuteurs basques qui ont rédigé le texte de 1435); le nom baracearte conserve au contraire sa phonétique basque, et même le hiatus plus tard effacé ;

- le même -e final roman atone a disparu dans garat, peut-être oyharat, et dans l'emprunt latin ospital (e);

- c'est un -a final, après une étape -e encore présente dans heguie (où -a était le déterminant basque) et echenique, qui a disparu dans urquiet, double du nom du village labourdin Urcuit, les deux lieux étant dits actuellement Urketa (effacement de la diphtongue) et qui procède d'un urki-eta, selon un modèle présent dans Viscarret, etc...; en revanche la forme pleine est dans haranbilleta, soroeta, oilloeta, berroeta...

La phonétique et la morphologie propres au basque reçoivent diverses réalisations graphiques. Certaines représentent des constantes très anciennes de la phonétique, comme l'alternance $-r /-n$ en finale du premier terme de composition qui donne oiharberro, oiharart(e), oiharat mais aussi oihanart(e) : la fréquence de la vibrante fait comprendre que 
la forme non composée régulière "oihan" a dû battre en brèche, par réfection corrective consciente ou non, la vieille phono-morphologie, et progressivement la supplanter, la langue oubliant ainsi ses propres procédés morphologiques, sans doute trop différents de ceux de la langue administrative. De même pour la chute des voyelles finales après vibrante de soro, sarri, larre, harri dans soriz, sorzabal, larzabal, argain, arregui, tandis qu'elles sont conservées dans soroeta, sarrigain (on trouve aussi ailleurs sarzabal, sarburu...), larragoien, harrieta. On peut comparer le nom hergaitz qui dénommait en 1366 l'une des deux maisons d'Ayherre dites simplement aguerre en 1435, Aguerre hergaytz, dont le premier terme est "herri" (à la rigueur une forme dissimilée de "harri" : sans cette dissimilation on aurait eu "hargaitz").

L'un des thèmes ordinaires de la phono-morphologie historique du basque est la variation en -a des voyelles finales -e et -o du premier terme de composé, ce qui fait à partir de bide, larre, otso, etxe les noms ici très réguliers et peut-être rectifiés (car des documents antérieurs ne donnent pas toujours la variante en -a pour les mêmes noms) : bidagayn, larragoyen, larrateguy, oxacellay, echavarren, echauazter, echapare, echavarne. Inversement le même mot "etxe" conserve la voyelle d'origine dans echeuerry, echeuerce, echeuehere, echegoyen. On doit s'interroger sur les raisons de ces variantes, qui font toucher du doigt toute la difficulté de la phonétique historique basque : variation de temps, la forme avec -a étant théoriquement la plus ancienne? formes assimilées phonétiquement, puisque les noms avec -a sont complétés par des termes ayant la même voyelle ouverte "barren, barne, bazter, -pare"? ou plutôt, car la force d'assimilation n'est valable ni pour "goien" ni pour "-tegi" ni pour "zelhai", tardivement dissimilées au contraire en raison des forces de correction et de réfection liées à un terme aussi présent que "etxe" dans la langue usuelle, ce qui semble la solution la plus cohérente (les formes avec-a pour etxaberri, etxagoien étant aussi bien attestées à époque ancienne, 1350,1366, 1412, que les formes "corrigées" comme bidegain, etc.) ? Traces de semi-isolats phonétiques, sous influence ou non du voisinage roman, comme cela est arrivé et arrive si souvent en phonétique basque ? Les faits sont là, la solution reste incertaine, même si les formes les plus cohérentes - on vient de voir qu'elles ne le sont pas toujours! - dans un même document doivent quelque chose et peut-être beaucoup au scribe lui-même et à ses compétences linguistiques. La maison dite en 1435 huhegun, qui doit être le porte-parole de la communauté d'Ayherre était nommée aussi en tête du fouage de 1366, sans forme de composition mais déterminée en Uhegona: le problème de la fermeture de -o devant nasale et les variations entre -o et -u dans cette position ("Bayonne" mais prononcé "Baioune" en gascon, "Ibarron", etc.) touchent un autre domaine classique des variations vocaliques en phonétique basque.

La graphie de larrando, écrit nettement la Rondo (c'est déjà l'anticipation du "français" La Rhune !) en 1366, et larrondo en 1412, forme normale du nom, montre une correction excessive ou une cacographie, peut-être par confusion avec des noms comme Larranda, Larrandaburu 
qui existent aussi : car la forme théorique larra-ondo doit aboutir par élimination du -a- en hiatus devant -o- au seul larrondo. Mais il n'y a guère, en phonétique basque comme ailleurs, de règle sans exception.

La forme echapare régulière dans cette lettre est le signe que le vieux composé etxa-gapare, graphie normale au XIV siècle et encore en 1412, a subi une "haplologie" ou chute de syllabe interne coutumière en phonétique basque, et documentée depuis le $\mathrm{X}^{\circ}$ siècle au moins dans l'oronyme bagibel, qui est certainement un baga-gibel, c'est-à-dire "derrière les hêtres". Le latinisme gapare issu d'un bas-latin "capale "principal" disparaît du vocabulaire usuel (bien qu'Oyhénart s'en souvienne encore deux siècles plus tard), et le sens ancien du nom est perdu.

S'agissant cette fois de pure morphologie, le texte de 1435 a l'avantage de fournir quelques exemples de noms de lieux déterminés avec "l'article" -a, ce qui du reste est fréquent, mais jamais régulier, dans les documents médiévaux de toutes époques. Les noms de maisons, qui sont encore toujours déterminés en basque, offrent probablement à cet égard les restes d'un usage très répandu, sinon général, aux époques les plus anciennes. Il en est resté de notables exemples comme Iruñ (e)a, Bizkaia, Zuberoa, etc. et Arberoa. Ici on lit lucua à Ayherre, maintenu sous cette forme jusqu'à aujourd'hui, et aussi garbissa à Saint-Martin, romanisé en Garbiçe en 1366. La finale basque, article ou non, est maintenue dans laharraga, çaualça, socobia, mais non pour çavaroz par comparaison au Sabarodsa de Laxague un demi-siècle plus tôt, qui donne l'ancien usage.

La consonne de transition dans les composés se lit deux fois : dans larrategui à Hélette, ainsi documenté depuis 1350, difficile à expliquer par l'élément "-(t)egi" pour "demeure de", puisque précisément ce type de composition est totalement inusité en Arbéroue encore en 1435. Il était présent et même assez abondant en Cize dès le XIV ${ }^{e}$ siècle, mais presque toujours sur des bases anthroponymiques, prénoms, surnoms ou noms de métiers. Les noms d'origine comme Baigorritegi, Jatsutegi, etc., rappelaient une vallée ou un village connus, alors que "larre" est un nom de maison très répandu. L'emplacement de la maison en écart et à flanc de colline indique sans hésitation un "larre" pour "lande", et l'on peut supposer tout au plus, puisque arregui existe aussi, et même dans les anthroponymes plus tardifs Larregui, une triple composition avec "larr-athe-hegi", soit "bord du passage de la lande". Le problème est comparable pour inhabartiri, où la dentale sépare vibrante et voyelle (comme elle le fait pour sifflante et voyelle dans lastiri, etc.), composition plus obscure et sûrement à trois termes, "ihi-nabar-iri" soit "domaine des joncs colorés", modèle par ailleurs répandu en toponymie ancienne; il est plus difficile, et peu probable à ce croisement de routes en plein hameau, de placer athe "porte, passage". Lélément final -ate, très vraisemblablement un vieux suffixe locatif variante de l'indéterminé -eta, plutôt que le mot précédent, réduit en phonétique romane à -at, se trouve dans le très répandu Garat(e) et aussi dans Oiharat, puisque "gareta" et "oihareta" ne sont jamais attestés. 
Des graphies particulières caractérisent la manière du scribe de 1435 : la latérale double - $l l$ - est presque systématique à l'intérieur des mots entre voyelles et n'indique donc nullement une "mouillure" : helleta, gellos, oxacellay, elliçalde, elliçayri, et il faut déduire qu'il n'y en a pas non plus, malgré des exemples modernes, dans haranbilleta, amezpilla, mendibil, tous composés avec bil "arrondi, groupé" et par extension "terrain arrondi, mamelon", à comparer aux graphies suivantes pour les mêmes maisons: ametzpil en 1366, harambilete en 1412, et de même oilloeta, actuellement sans mouillure. La séquence automatique -mb- en graphie romane commune dans ces listes est évitée lorsque c'est la séquence -nb- qui est étymologique, comme dans les composés de (h)aran "vallée" haranbilleta, haranburu.

Les sifflantes dorso-alvéolaires sont sans équivoque avec $\xi$ ou z dans çaualça, çavaroz, yzturiz: la forme moderne ist- est, dans les graphies qui se veulent basques de ce nom, altérće à partir de la graphie romane. Mais $s$ ou ss peuvent aussi transcrire la dorso-alvéolaire, et non l'apico-alvéolaire basque écrite aujourd'hui $s$, dans haristoy, garbissa, alors que l'apico-alvéolaire est sûre dans les composés de sarri et soro, mais très incertaine dans issuri, moderne "Ixuri", où la palatalisée "chuintante" est pourtant improbable, puisque le texte la transcrit toujours par ch dans eche-, miche.

Le cas unique de oxacellay indique certainement une affriquée apicale, moderne " $t \mathrm{~s}$ ", comme le suggèrent la tradition du radical otso (c'est précisément "la plaine des loups") et la graphie ossaçelay de 1412. En dehors de cet exemple, et de ch qui en prononciation castillane correspond toujours à $t c h$ (graphie systématique dans le fouage gascon de 1412) dans eche- et sans doute miche, le texte ne permet de lire aucune affriquée " $\mathrm{tz}$ ", " $\mathrm{ts}$ " ou " $\mathrm{tx}$ ": londayz, ypuzaguerre, ganderaz, yzturiz, soriz, aynci,-uerce. Mais les affriquées, beaucoup plus rares dans les textes médiévaux et classiques, surtout après liquide et nasale, ont proliféré dans certains dialectes modernes, au point de paraître aujourd'hui, très abusivement et dommageablement pour l'équilibre phonétique et l'euphonie de la langue, comme des caractéristiques propres à celle-ci.

La régularité des aspirations et consonnes aspirées étonne dans un texte navarro-castillan issu de la chancellerie navarraise, puisque l'un des traits dialectaux les plus anciens et les plus connus du basque navarrais est d'avoir perdu très tôt l'aspiration au contact du roman aragonais. L'enquête navarro-castillane de 1350 l'élimine presque systématiquement, tandis que les listes de 1366 et 1412 rédigées en gascon les reproduisent. Il en est de même ici, avec même quelque abus dans huhegun par rapport au Uhegona de 1366, tant à l'initiale dans helleta (en 1350 elete), heguie, haristoy, haranburu..., qu'entre voyelles dans ayherre (en 1350 Ayerre, à comparer à l'aragonais Ayerbe de même étymologie), uharte, uhalde, lohigorri, plus rarement en consonne aspirée dans inhabartiri. Le texte se conforme à la prononciation locale, sans reproduire pourtant, comme d'autres écrits, toutes les aspirations dont on sait que les dialectes basques de France les ont considérablement étendues. À la différence de harrieta, les noms argain, arregi ont 
évacué partout l'aspiration initiale peut-être par dissimilation ancienne devant $-g$ -

L'étymologie de quelques noms mérite réflexion. Et tout d'abord celle d'Uhegon (VHAGVN au linteau de la maison "neuve" de 1760), voisine de Barne-uharte dans une boucle de l'Arbéroue en limite de Hasparren, sur la route de La Bastide-Clairence, ville bâtie au début du $\mathrm{XIV}^{\mathrm{e}}$ siècle sur les landes communes d'Arbéroue. Est-ce un composé de ur "eau" comme le voisin uharte? Le mot de même forme uhagun "écume d'eau" étant bien improbable pour nommer un très ancien domaine limitrophe, il faut sans doute penser soit au terme uhe/uha "perche, bâton" qui a pu convenir à un lieu boisé d'usage particulier (ailleurs eihar ou galhar/garhar nomment de nombreux lieux, hameaux et maisons, où se ramassait le "bois mort"), puisque la maison est à la fois en bord de rivière et au début des anciennes landes d'Arbéroue, soit au quasi-homonyme uher "gris sombre, trouble", notion de couleur et de lumière convenant au site, et qui serait alors l'inverse sémantique de la maison de Saint-Martin Arguioz sur la base argi "lumière", le sémantisme des notions de "lumineux" et "sombre" étant aussi proche du radical garbi "propre, net, clair" de la maison Garbitz du même lieu. Le groupement des notions de même nature ou complémentaires est un trait connu de la vieille toponymie.

Le nom Oilloeta ne devrait pas faire allusion, d'après les caractères généraux des anciens noms de lieux, à un "lieu de poules", pas plus que la plupart des toponymes anciens de même radical, et ce peut être l'altération d'un primitif + oloeta "lieu d'avoine sauvage", plus conforme à la thématique habituelle; il faut reconnaître cependant que l'apparition de ce nom seulement en 1435 plaide pour une exception. Dans Sendugarat le premier élément rappelle seulement l'ancien emprunt sendo "sain, guéri" et par extension "fort", assez employé comme surnom au Moyen Àge (Garcia sendoa 1110), mais son emploi ici reste mystérieux et lié à des circonstances difficiles à imaginer.

Irurita est le locatif de Iruri "trois villes", c'est-à-dire "trois domaines", et ici "lieu des trois domaines". Comme c'est aussi avec le même sens le nom du village du Baztan, vallée du royaume proche de la Basse-Navarre, on ne sait s'il faut le prendre au sens propre, la maison étant déjà citée en 1388 , ou comme importation d'un toponyme extérieur, comme cela se pratiquait aussi exceptionnellement pour des noms de maisons en Soule, et aussi dès le XIII' siècle à Valcarlos.

Le cas de Minche, puisque l'inscription de 1721 Minge et la forme actuelle Minchia indiquent que le copiste de 1435 a dû oublier la marque de la nasale ou que celle-ci s'est effacée, reste problématique : aucun nom médiéval, pami les milliers recensés, ne donne une forme comparable. Les termes qui la rappellent comme min- "aigreur", son dérivé minda "purin" sont peu utiles pour la toponymie, et les radicaux toponymiques connus comme l'emprunt mina/miña "vigne" et min"plant" s'articulent mal avec la finale, à moins que le nom ait subi une altération peu explicable. Phonétiquement minche et surtout minge 
suggèrent un nom roman : très curieusement le fouage béarnais de 1385 donne des noms comparables de forme Mindaa que M. Grosclaude tient pour "obscurs" (Dictionnaire étymologique des noms de famille gascons, p. 179, Pau 1992). L'un des diminutifs de "Dominique", attestés sous des formes voisines, serait bien l'unique exemple de ce type pour un nom de maison en Arbéroue à cette date.

Deux noms présentent des formes curieusement apparentées : elçurren à Saint-Martin, et urrelçe à Hélette, actuellement altéré en Urbeltz, mais les témoignages anciens et les noms d'état civil issus de cette maison, notaires et autres, portent toujours D(')Urrels. La tentation est grande d'y voir quelque légendaire "pot" ou "soupière d'or", et il semble que l'on n'ait pas manqué d'y succomber quelque peu. Pour elçurren, des graphies comme Alzurren au XII' siècle, Alzurrun au XIV ${ }^{e}$, actuel Elzurrun suggèrent une altération de *(h)altz-urrun au sens approximatif de "de l'autre côté des aulnes" conforme aussi à des éléments topographiques locaux. Il se peut alors que le nom de maison de Hélette, dont le premier élément constant urr-exclut $u r$ "eau", en soit simplement une forme inversée de type commun en toponymie ancienne, issue d'un ancien *urr $(u)-(h)$ altz avec fermeture vocalique entraînée par la voyelle initiale. De tels changements prouveraient que les sens primitfs de ces toponymes se sont perdus, phénomène fréquent, ouvrant la voie aux réfections, sémantiques, analogiques, phonétiques, de diverses sortes.

Parmi les altérations récentes subies pas les noms médiévaux, des métathèses phonétiques s'observent : pour Argiotz devenu Argoitz, pour Sarrilbe, de difficile analyse (-l-représente-t-il ale ou ele, ou autre chose ?), passé à Sarrible, avec un groupe -bl-totalement étranger à la phonétique basque. Le gortayri de Hélette s'est changé, comme partout dans la région, en un plus compréhensible Bortairi : mais les vieux toponymes désignaient toujours la "cour" (bas-latin curte) et non la "porte".

\section{Laburpena}

Arberoako 128 etxe, haien orduko etxeko-jaun eta hauen ondoriotasunezko jabe guziak betiereko noble edo aitorenseme egin zituen Jean II eta Blanca Nabarrako errege eta erreginaren eskutitz ideki batek 1435-an. Ixtorioko liburu batzuek aitzineko mendean (Yanguas y Miranda, P. Haristoy...) eskutitz hori aipatu dute, erranez 111 etxe nobletzen zirela: 111 jaun izendatzen baldin badira, eta ere hetan bortz etxekandere, gauza berenaz ohargarria, etxeak berak 128 dira, oro jadanik "infanzun" ekarriak ziren etxe zaharretarik bertze. Eskutitz hori emaiten da osoan. Ohartzen da aitorensemetze hori Arberoako laboretxetako jaunek berek zutela galdegiten, eta erregek onhartzen zuela erresumako mugan zirelako estakuruan : alabainan ehun urteko gerlaren azken hamarkadak dira, Nabarra Frantziaren alde, Lapurdi Angeleterrako erregeren eskuan... 
Ixtorioko gaietan urrunago aitzinatu gabe, hau ixtoriatzailen egitekoa izanez, ikertzen dira jende eta leku izenak. Jadanik ezagunak ziren aro zaharragokoekin gomparatuz leku beretan, ikusten da lehenik nola aldatu diren jende izenak eta aldatzen ari hamabortzgarren mende erditara, batzu ahanzten edo bekantzen, bertze batzu usutzen, berri zenbait agertzen. Etxe izenetan, badira aitzineko dokumentoek, XII-XIII-XIV mendetan, aipatzen etzituztenak, etxe berriak menturaz. Idazkiak gehienean, idazleari zor zaizkion berezitasun batzu salbu, bereziki zuzen emaiten ditu, ala ahoskeraren aldetik ala morfologiaren. Ikertzen dira azkenik zerrenda hortaraino ezagunak etziren edo Arberoako aldean baizik agertzen eztiren izen bakar batzu, eta izen batzuek geroztik ukan dituzten aldaketa zenbait.

Jean-Baptiste ORPUSTAN

Université Michel de Montaigne - Bordeaux III ERS 142 du CNRS 